\title{
Ecología trófica del búho terrestre Athene cunicularia punensis (Strigiformes: Strigidae) en el archipiélago de Jambelí, provincia de EI Oro, suroeste de Ecuador
}

\section{Feeding ecology of the Burrowing Owl Athene cunicularia punensis (Strigiformes: Strigidae) in the Jambelí archipelago, El Oro province, southwestern Ecuador}

\section{Adrian Orihuela-Torres ${ }^{1 *}$, Leonardo Ordóñez-Delgado ${ }^{1}$, Jorge Brito ${ }^{2,3}$, Fausto López ${ }^{1}$, Marina Mazón ${ }^{4,5} y$ Juan F. Freile ${ }^{6}$}

1 Departamento de Ciencias Biológicas. Universidad Técnica Particular de Loja. CP: 11-01-608. Loja, Ecuador.

2 Instituto Nacional de Biodiversidad. Calle Rumipamba 341 y Av. de los Shyris. Casilla: 17-07-8976. Quito, Ecuador.

3 Instituto de Ciencias Biológicas, Escuela Politécnica Nacional. PO Box: 17-01-2759, Quito, Ecuador.

4 Programa de Investigación en Biodiversidad y Servicios Ecosistémicos, Universidad Nacional de Loja, Ciudadela Universitaria, sector La Argelia, EC 110101 Loja, Ecuador.

5 Departamento de Ciencias Ambientales y Recursos Naturales / Instituto de Investigación de Biodiversidad CIBIO (Centro Iberoamericano de Biodiversidad). Universidad de Alicante. Apdo. Corr. 99, 03080. Alicante, España.

6 Comité Ecuatoriano de Registros Ornitológicos. Pasaje El Moro E4-216 y Norberto Salazar, EC 170184, Tumbaco, Ecuador.

*Autor para correspondencia

Email Adrian Orihuela-Torres: adrian.orihuela89@gmail.com

Email Leonardo Ordoñez-Delgado: lyordonez2@utpl.edu.ec

Email Jorge Brito: jorge.brito@ambiente.gob.ec

Email Fausto López: fvlopezx@utpl.edu.ec

Email Marina Mazón: marinamazonmor@gmail.com

Email Juan F. Freile: jfreileo@yahoo.com

\section{Resumen}

Estudiamos la dieta del búho terrestre Athene cunicularia punensis en cuatro territorios dentro del archipiélago de Jambelí, provincia de El Oro, suroeste de Ecuador, entre noviembre de 2015 y abril de 2016, mediante la recolección y análisis de 182 egagrópilas. Los artrópodos constituyeron el principal grupo-presa en frecuencia de aparición (86.5\%), seguido por los mamíferos (12\%) y las aves (1.5\%). En cuanto a biomasa consumida, los mamíferos (Mus musculus y Rattus rattus) aportaron $58.5 \%$, los artrópodos $33.2 \%$ y las aves $8.2 \%$. No encontramos diferencias significativas de la dieta entre las cuatro parejas estudiadas. En este trabajo se confirmó el rol de Athene cunicularia como un importante depredador de plagas como roedores introducidos y artrópodos potencialmente dañinos.

Palabras clave: Athene cunicularia punensis; Archipiélago de Jambelí; dieta; egagrópilas; presas.

\section{Abstract}

The diet of Burrowing Owl (Athene cunicularia punensis) was studied in four territories in the Jambelí archipelago, El Oro, southwestern Ecuador, from November 2015 to April 2016, collecting 182 pellets. Arthropods were the main prey group in frequency of occurrence $(86.5 \%)$, followed by mammals (12\%) and birds (1.5\%), but mammals (Mus musculus and Rattus rattus) contributed $58.5 \%$ of the biomass, arthropods $33.2 \%$ and birds $8.2 \%$. No significant differences were found in the diet among the four pairs studied. Our study confirmed the important role of Athene cunicularia as predator of pests like introduced rodents and potentially harmful arthropods.

Keywords: Athene cunicularia punensis; archipelago of Jambelí; diet; pellets; prey.

Citación:

Orihuela-Torres A., L. Ordóñez-Delgado, J. Brito, F. López, M. Mazón y J.F. Freile. 2018. Ecología trófica del búho terrestre Athene cunicularia punensis en el archipiélago de Jambelí, provincia de El Oro, suroeste de Ecuador. Revista peruana de biología 25(2): 123 - 130 (Mayo 2018) doi: http://dx.doi.org/10.15381/rpb.v25i2.13376

$\begin{array}{ll}\text { Presentado: } & 22 / 06 / 2017 \\ \text { Aceptado: } & 02 / 02 / 2018\end{array}$

Publicado online: 30/05/2018
Fuentes de financiamiento: Este trabajo se elaboró dentro del proyecto "Evaluación de la efectividad de manejo y estado de conservación de áreas de manglar de la provincia de El Oro, Código: PROY_CCNN_1070; financiado por la UTPL a través de la VI convocatoria internacional de proyectos, modalidad "Proyectos Semilleros".

Información sobre los autores: AOT: Trabajo de campo y laboratorio, análisis de datos, redacción de metodología, resultados y discusión; LOD: Trabajo de campo, redacción de resultados y discusión; JB: Identificación de los restos de mamíferos, redacción y revisión del manuscrito; MM: Identificación de artrópodos, redacción y revisión del manuscrito, FL: Redacción y revisión del manuscrito; JFF: Redacción y revisión detallada de todo el documento.

Los autores no incurren en conflicto de intereses. 


\section{Introducción}

La especialización trófica entre las aves deriva de su historia evolutiva y de los factores ecológicos en los cuales se desarrollan. Existe una correlación entre distintos aspectos morfológicos de las aves (i.e., forma del pico, tamaño de las patas), etológico (i.e., tipo de vuelo, comportamiento de forrajeo) y ecológico (i.e., micro-hábitat de alimentación) con su dieta. Así, los depredadores grandes tienden a capturar presas de mayor volumen que los depredadores pequeńos, o los depredadores que cazan en vuelo tienden a capturar presas más móviles que aquellos que atacan desde una percha (Greene 1986).

Entre las aves rapaces nocturnas (Strigiformes), hay taxones con mayor especialización en depredar vertebrados (e.g., roedores en Asio flammeus y Tyto alba; Clark 1975, Rocha et al. 2011) o invertebrados (e.g. varias especies de Megascops; König $\&$ Weick 2008). No obstante, muchos Strigiformes pueden adaptarse a explotar presas que se tornan súper abundantes estacional, local o estocásticamente (Lack 1946, Silva-Porto \& Cerqueira 1990, Silva et al. 1995). Esto indica que la dieta de una especie puede relacionarse con la disponibilidad de presas más que con una predilección por presas específicas (Kavanagh 2002), aunque con diferencias interespecíficas según el tamaño del depredador o las tácticas de forrajeo (Greene 1986). Por ejemplo, Martínez et al. (1998) encontraron una mayor cantidad de aves e insectos en la dieta de $A$. flammeus ante la escasa disponibilidad de roedores.

Muchas aves de presa de amplia distribución geográfica, que ocupan una gran variedad de hábitats en sus áreas de distribución, tienen gran plasticidad en sus hábitos alimenticios (Rocha et al. 2011). Athene cunicularia (Molina, 1782) es un búho pequeño de dieta generalista que habita en zonas abiertas, generalmente áridas, desde el centro-norte de Estados Unidos hasta la Patagonia Argentina (König \& Weick 2008). Es un búho sedentario, parcialmente diurno, que tolera niveles considerables de alteración antropogénica de sus hábitats. En buena medida, su capacidad para adaptarse a la disponibilidad de presas según su abundancia o escasez, influye en su capacidad para resistir cambios drásticos de hábitat (Kavanagh 2002).

Su dieta en general comprende mayor cantidad de artrópodos, pero mayor biomasa de roedores (Marti 1974, Jaksic \& Marti 1981, Motta-Junior \& Bueno 2004, Moulton et al. 2005, Cadena-Ortiz et al. 2016), con variaciones según la abundancia y accesibilidad de presas (Vieira \& Texeira 2008), según su comportamiento de cacería (Bueno \& Motta Junior 2008) y de acuerdo a las relaciones interespecíficas dentro de una comunidad de aves nocturnas (Motta-Junior 2006).

Existe abundante información sobre la ecología trófica de A. cunicularia en Brasil, (Motta-Junior \& Bueno 2004), Chile (Carevic et al. 2013, Cruz-Jofré \& Vilina 2014), Perú (Medina et al. 2013), Argentina (Pardiñas \& Cirignoli 2002, Nabte 2004, Sánchez et al. 2008), Paraguay (Andrade et al. 2004), República Dominicana (Wiley 1998), México (Rodríguez-Estrella 1997)y especialmente Estados Unidos (Moulton et al. 2005, Hall et al. 2009, Bielefeld 2014), pero muy poco en Ecuador, en donde los estudios de dieta de búhos apenas están iniciándose (CadenaOrtiz et al. 2016).

Ecuador alberga a las subespecies $A$. cunicularia pichinchae (Boetticher 1929) que se distribuye en la región Andina y $A$. cunicularia punensis (Chapman, 1914) que se distribuye en la costa centro sur de Ecuador y noroeste de Perú (Holt et al. 2017). Actualmente, solo existe un estudio que analiza la ecología trófica de la especie para Ecuador (Cadena-Ortiz et al. 2016), el mismo que corresponde a la subespecie $A$. cunicularia pichinchae, pero hasta donde conocemos, no se cuenta con información publicada de la ecología trófica de la subespecie $A$. cunicularia punensis en Ecuador y solo hay un estudio en Perú (Medina et al. 2013).

Las egagrópilas son una herramienta ampliamente utilizada para determinar la dieta de aves rapaces diurnas y nocturnas, ardeidas y córvidos, entre otros (Marti 1987). Conocer la dieta de una especie sirve para determinar, entre otros aspectos, sus relaciones intra e interespecíficas (Marti et al. 1993). Por ello, en este trabajo presentamos datos de su alimentación en dos islas muy próximas al territorio continental del suroccidente de Ecuador, como resultado de la colecta y análisis de egagrópilas en cuatro territorios reproductivos de la especie. Comparamos nuestros resultados entre las cuatro parejas estudiadas con respecto a cantidad de presas, aporte energético y amplitud trófica, y analizamos los datos a la luz del conocimiento sobre la ecología trófica en A. cunicularia en Ecuador y en el Neotrópico.

\section{Material y métodos}

Área de estudio.- El archipiélago de Jambelí, se ubica en el suroeste de Ecuador, en la provincia de El Oro (Fig. 1). Ocupa aproximadamente 41700 ha y se compone de 18 islas, en cinco de las cuales habitan comunidades humanas de manera permanente: Bellavista, Costa Rica, Pongalillo, Las Huacas y Las Casitas. Este archipiélago se considera como un Área de Importancia para la Conservación de Aves (IBA, por sus siglas en inglés; Freile \& Santander 2005). En noviembre de 2015 se localizaron tres parejas de $A$. cunicularia punensis (Pareja 1, 2 y 3), con sus respectivos nidos, en los alrededores de la comunidad Las Casitas $\left(3^{\circ} 22^{\prime} 33^{\prime \prime} \mathrm{S}, 80^{\circ} 07^{\prime} 52^{\prime \prime} \mathrm{W}\right)$; y una pareja adicional (Pareja 4) en la comunidad Las Huacas (3²0'59" S, 8009'50'W).

El hábitat en los territorios estudiados corresponde a un arbustal deciduo en zonas adyacentes a playas de arena o playas rocosas (Cerón 2013); su vegetación es achaparrada, de 2-4 m de altura, y está compuesta por plantas herbáceas, rastreras o trepadoras, como Cryptocarpus pyriformis, Hippomane mancinella, Scutia spicata, Vallesia glabra, Batis maritima, Canavalia maritima, Ipomoea pescaprae y Sesuvium portulacastrum (Cerón et al. 1999, Cerón 2013). Los territorios con nidos están adyacentes a centros poblados que presentan fuerte intervención humana.

Toma de muestras.- En nuestro estudio recolectamos egagrópilas frente a cada uno de los nidos estudiados y posteriormente las analizamos.

Entre noviembre de 2015 y abril 2016 colectamos egagrópilas en los nidos y posaderos de las cuatro parejas de $A$. cunicularia punensis cada dos meses. Envolvimos cada egagrópila en papel aluminio al momento de la colecta de las muestras para mantenerlas separadas y las colocamos en fundas plásticas de cierre hermético con un código de campo asignado a cada una de ellas para su correcta identificación.

En laboratorio, medimos la longitud y ancho de cada egagrópila utilizando un calibrador digital Stainless Hardened (precisión $0.01 \mathrm{~mm}$ ) y el peso en seco utilizando una balanza de precisión Sartorius LA-230P. Además, diseccionamos en seco cada egagrópila y colocamos los elementos del contenido 


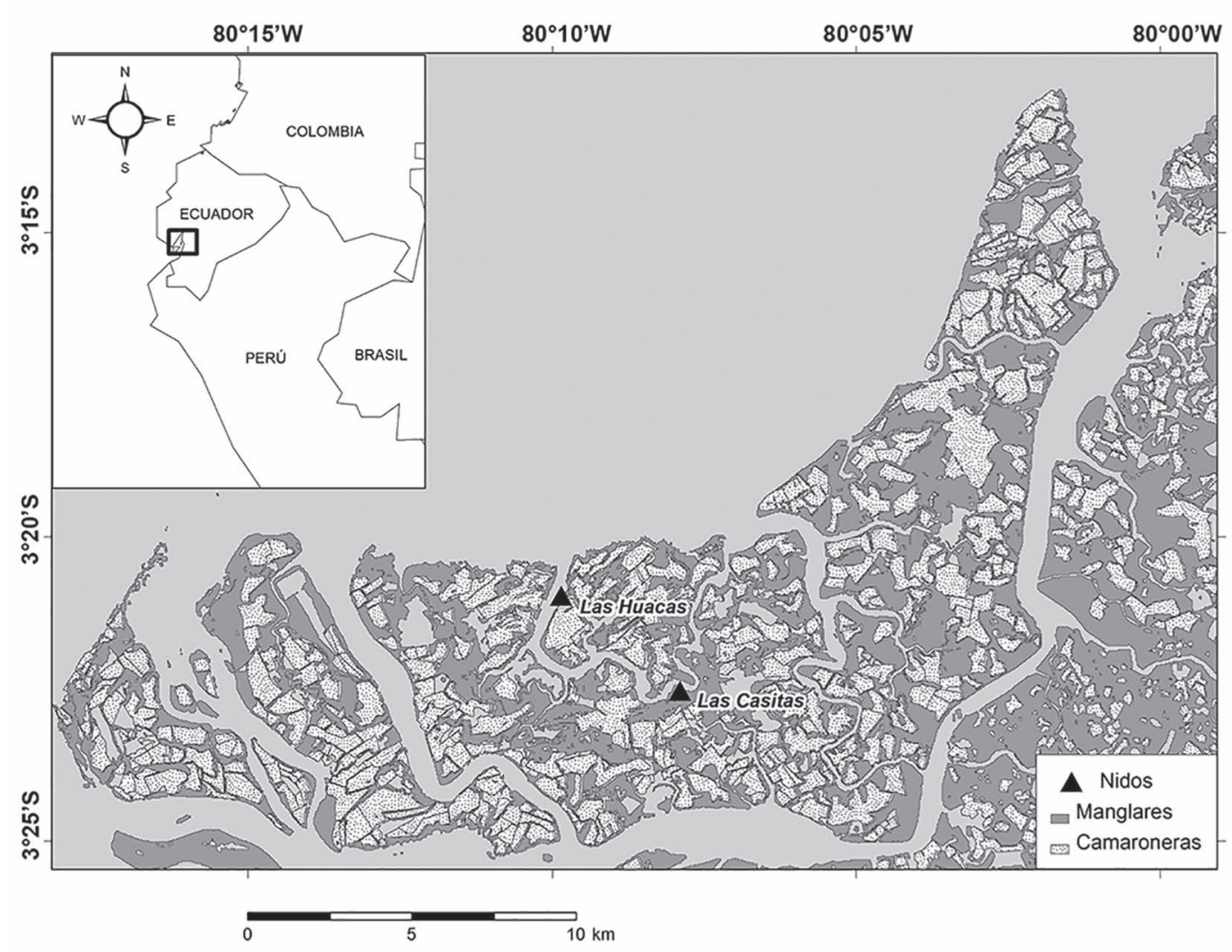

Figura 1. Archipiélago de Jambelí, El Oro, suroeste de Ecuador. En gris oscuro se puede observar la vegetación de manglar y en gris claro las camaroneras. Los puntos hacen referencia a las dos islas donde se encuentran los territorios de Athene cunicularia punensis estudiados.

en placas Petri para su identificación y cuantificación. Los contenidos disgregados de las egagrópilas fueron depositados en el Museo de Vertebrados de la Universidad Técnica Particular de Loja (MUTPL).

Análisis del contenido de las egagrópilas.- Para el análisis de egagrópilas, primero las disgregamos y guardamos los restos de las presas en fundas herméticas, separadas y numeradas. Posteriormente, separamos las muestras por grupos taxonómicos (aves, mamíferos, insectos, arácnidos y malacostráceos).

Los restos de artrópodos fueron separados, cuantificados e identificados hasta el nivel de orden. Los coleópteros se identificaron a nivel de familias comparándolos con especímenes en la colección del Laboratorio de Sanidad Vegetal del Área Agropecuaria y Recursos Naturales Renovables de la Universidad Nacional de Loja y del MUTPL. Para la cuantificación, contabilizamos el número de partes reconocibles de los ejemplares encontrados: telson en escorpiones, cabezas o pronotos en coleópteros y cabezas en himenópteros y ortópteros. A partir de los fragmentos, estimamos el peso de los artrópodos basados en Carevic et al. (2013) y Cruz-Jofré \& Vilina (2014).

La identificación de mamíferos se realizó a nivel de especies, a partir de las mandíbulas. Para la obtención de su peso promedio utilizamos la colección de mastozoología del Museo Ecuatoriano de Ciencias Naturales (MECN), Quito. La identificación de aves no llegó a nivel de especies, ya que entre los restos no encontramos plumas o cráneos enteros, apenas fragmentos y huesecillos disgregados. El peso de las aves se estimó en base al tamaño de los huesecillos, que se aproximaban a los de un paserino de tamaño medio (Morales 2016).

Análisis de datos.- Para cada grupo taxonómico y cada presa calculamos el porcentaje de frecuencia de aparición $(f a)$ y el porcentaje de biomasa consumida $(b)$. La frecuencia de aparición $(f a)$ se calculó como el número de individuos de una especie $(n)$ dividido entre el total de individuos $(n)$ y multiplicado por cien.

$$
f a=\frac{n}{n_{t}} * 100
$$

El porcentaje de biomasa (b) se calculó multiplicando el número de individuos de una especie $(n)$ por su peso $(p)$, dividido entre el peso de todas las presas $(p)$ y multiplicado por cien (Rau 2000).

$$
b=\frac{n * p}{p_{t}} * 100
$$

Además, calculamos la amplitud trófica mediante el índice de Levins (B), el cual propone que la amplitud puede ser estimada a partir de la uniformidad de la distribución de los individuos entre los diversos recursos alimenticios (Krebs 1989). Este índice se utilizó para cada pareja de $A$. cunicularia punensis individualmente, y para todas las parejas estudiadas en conjunto, mediante la siguiente fórmula:

$$
\mathrm{B}=\frac{1}{\sum p j^{2}}
$$

Donde $p j$ es la proporción de cada ítem presa en la dieta. Los valores obtenidos fueron estandarizados (BA) con el método 
de Hurlbert (1978), para ser expresados en una escala de 0 a 1 , mediante la siguiente fórmula:

$$
\mathrm{BA}=\frac{B-1}{n-1}
$$

En este caso, n es número de ítems presa. Este índice de amplitud trófica es mayor cuando las frecuencias de utilización son las mismas para cada uno de los recursos consumidos y menor cuando el recurso consumido es uno solo; es decir, cuando es un especialista estricto (Krebs 1989).

Para determinar las diferencias entre la dieta de las parejas se utilizó la prueba de Kruskal-Wallis, ya que mediante el test de Shapiro-wilk, se corroboró que los datos no presentaban una distribución normal. Estos análisis se realizaron utilizando el software estadístico R (R Core Team 2016).

\section{Resultados}

Colectamos y analizamos 182 egagrópilas provenientes de los cuatro nidos estudiados, en las cuales encontramos 1264 presas (media $=316 ; \mathrm{SD}=116.31$; rango $=175-418$ ). Tomamos medidas de longitud, ancho y peso de las egagrópilas, con los siguientes resultados: longitud (media $=26.31 \mathrm{~mm} ; \mathrm{SD}=8.64$ $\mathrm{mm}$; rango $=13.78-58.14 \mathrm{~mm})$; ancho $($ media $=13 \mathrm{~mm} ; \mathrm{SD}=$ $2.24 \mathrm{~mm}$; rango $=8.73-19.96 \mathrm{~mm})$; peso $($ media $=1.14 \mathrm{~g} ; \mathrm{SD}=$ $0.64 \mathrm{~g} ;$ rango $=0.3-3.88 \mathrm{~g})$.
Aunque la mayoría de presas no fueron identificadas a nivel de especie, encontramos al menos 30 taxones diferentes, pertenecientes a cinco clases taxonómicas: Aves, Mammalia, Insecta, Arachnida y Malacostraca (Tabla 1). El tamaño de las presas varió desde $1 \mathrm{~g}$ (Formicidae), hasta $162 \mathrm{~g}$ (Rattus rattus). Además de estas presas, en algunas egagrópilas aparecieron restos de gasterópodos, escamas de pez y escamas de una serpiente de la familia Colubridae. También registramos restos de Zenaida meloda y Muscigralla brevicauda a la entrada de la madriguera de dos de los nidos estudiados, y cabezas de Rhinella horribilis en la entrada de todas las madrigueras (Fig. 2), sin embargo en las egagrópilas no encontramos restos de esta especie. En la entrada de todas las madrigueras también se encontró acumulación de restos vegetales (semillas de Terminalia catappa) y excrementos, posiblemente de Lycalopex sechurae y de perros domésticos (Fig. 3).

Los artrópodos fueron el principal grupo presa en frecuencia de aparición, seguido por los mamíferos y las aves (Tabla 1). A nivel de orden, destacan por su frecuencia los coleópteros y ortópteros (Tabla 1). La frecuencia de aparición de artrópodos fue alta en todas las parejas, pero variable entre ellas (Tabla 2). La pareja 4 (Las Huacas) tuvo el mayor porcentaje en frecuencia de aparición de mamíferos y aves (Tabla 2).

Tabla 1. Composición de la dieta de Athene cunicularia punensis en cuatro territorios estudiados en el archipiélago de Jambelí, provincia de El Oro, suroeste de Ecuador. Se muestra el número de individuos que aparecen en las egagrópilas ( $\mathrm{N}^{\circ}$ individuos), el porcentaje de la frecuencia de aparición $(f a)$, la biomasa total en gramos (Peso $(\mathrm{g})$ ) y en porcentaje $(b)$.

\begin{tabular}{|c|c|c|c|c|}
\hline & $\mathbf{N}^{0}$ individuos & $f a$ & Peso (g) & $b$ \\
\hline \multicolumn{5}{|l|}{ Rodentia } \\
\hline \multicolumn{5}{|l|}{ Muridae } \\
\hline Mus musculus & 140 & 11.1 & 1960 & 30.6 \\
\hline Rattus rattus & 11 & 0.9 & 1782 & 27.9 \\
\hline TOTAL MAMMALIA & 151 & 12 & 3742 & 58.5 \\
\hline Passeriformes & 19 & 1.5 & 524.4 & 8.2 \\
\hline TOTAL AVES & 19 & 1.5 & 524.4 & 8.2 \\
\hline \multicolumn{5}{|l|}{ Coleoptera } \\
\hline Tenebrionidae & 545 & 43.1 & 545 & 8.5 \\
\hline Cerambycidae & 9 & 0.7 & 19 & 0.3 \\
\hline Scarabaeidae & 28 & 2.2 & 56 & 0.9 \\
\hline Curculionidae & 2 & 0.2 & 4 & 0.1 \\
\hline no identificados & 9 & 0.7 & 18 & 0.3 \\
\hline \multicolumn{5}{|l|}{ Orthoptera } \\
\hline no identificados & 281 & 22.2 & 566 & 8.8 \\
\hline \multicolumn{5}{|l|}{ Hymenoptera } \\
\hline Formicidae & 9 & 0.7 & 9 & 0.1 \\
\hline Otras familias (no id.) & 1 & 0.1 & 1 & 0 \\
\hline \multicolumn{5}{|l|}{ Dictyoptera } \\
\hline Blattidae & 45 & 3.6 & 90 & 1.4 \\
\hline TOTAL INSECTA & 929 & 73.5 & 1308 & 20.5 \\
\hline \multicolumn{5}{|l|}{ Scorpionida } \\
\hline no identificadas & 99 & 7.8 & 495 & 7.7 \\
\hline \multicolumn{5}{|l|}{ Araneae } \\
\hline no identificadas & 62 & 4.9 & 310 & 4.8 \\
\hline TOTAL ARACHNIDA & 161 & 12.7 & 805 & 12.5 \\
\hline \multicolumn{5}{|l|}{ Decapoda } \\
\hline no identificadas & 4 & 0.3 & 20 & 0.3 \\
\hline TOTAL MALACOSTRACA & 4 & 0.3 & 20 & 0.3 \\
\hline
\end{tabular}




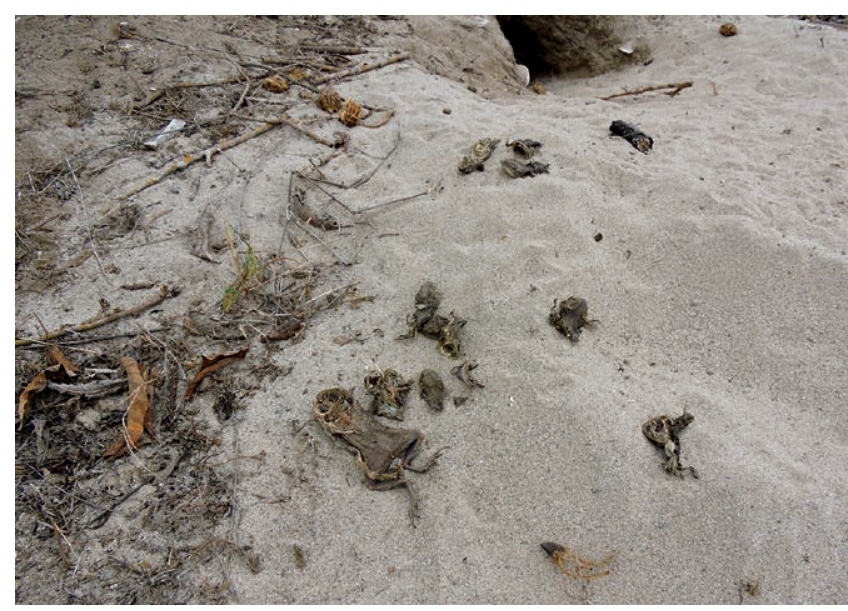

Figura 2. Restos de Rhinella horribilis en la entrada de una de las madrigueras de Athene cunicularia punensis en el archipiélago de Jambelí, El Oro, suroeste de Ecuador. Se observa como no se comen la cabeza y la piel, debido a que es donde se sitúan las partes tóxicas.

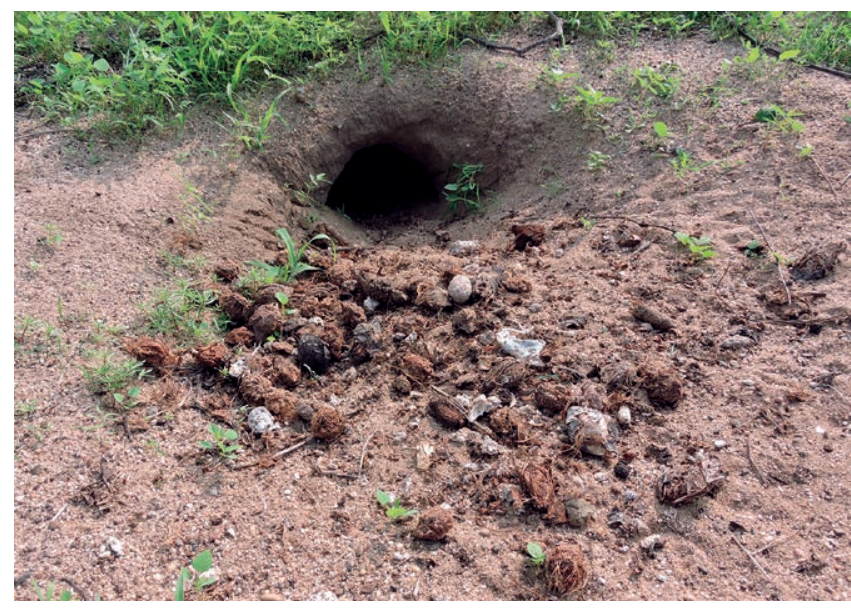

Figura 3. Nido de Athene cunicularia punensis en el archipiélago de Jambelí, El Oro, suroeste de Ecuador. Se observan frutos de Terminalia catapa y excrementos de cánidos en la entrada para confundir a los depredadores con el olor de esta.

Tabla 2. Composición de la dieta de las cuatro parejas estudiadas de Athene cunicularia punensis en el archipiélago de Jambelí, El Oro, suroeste de Ecuador. En los resultados se muestran el porcentaje de frecuencia de aparición (fa) y el porcentaje de biomasa consumida $(b)$ de cada presa, por pareja.

\begin{tabular}{|c|c|c|c|c|c|c|c|c|}
\hline & \multicolumn{2}{|c|}{ P1 } & \multicolumn{2}{|c|}{ P2 } & \multicolumn{2}{|c|}{ P3 } & \multicolumn{2}{|c|}{ P4 } \\
\hline & $f a$ & $b$ & $f a$ & $b$ & $f a$ & $b$ & $f a$ & $b$ \\
\hline \multicolumn{9}{|l|}{ Rodentia } \\
\hline \multicolumn{9}{|l|}{ Muridae } \\
\hline Mus musculus & 9.2 & 36.4 & 3.4 & 7.2 & 9.7 & 21 & 19 & 52.1 \\
\hline Rattus rattus & 0.2 & 10 & 2.3 & 55.8 & 2 & 50.4 & 0 & 0 \\
\hline TOTAL MAMMALIA & 9.4 & 46.4 & 5.7 & 63.1 & 11.7 & 71.4 & 19 & 52.1 \\
\hline Passeriformes & 0.7 & 5.1 & 2.3 & 9.5 & 1.7 & 7.2 & 2.1 & 11.4 \\
\hline TOTAL AVES & 0.7 & 5.1 & 2.3 & 9.5 & 1.7 & 7.2 & 2.1 & 11.4 \\
\hline \multicolumn{9}{|l|}{ Coleoptera } \\
\hline Tenebrionidae & 55.2 & 15.7 & 35.8 & 5.4 & 50.5 & 7.8 & 23.8 & 4.7 \\
\hline Cerambycidae & 1.3 & 0.8 & 1.3 & 0.3 & 0.3 & 0.1 & 0 & 0 \\
\hline Scarabaeidae & 0 & 0 & 0 & 0 & 8.7 & 2.7 & 0.6 & 0.2 \\
\hline Curculionidae & 0 & 0 & 0 & 0 & 0.7 & 0.2 & 0 & 0 \\
\hline no identificados & 0 & 0 & 0.3 & 1.1 & 0.3 & 0.1 & 0 & 0 \\
\hline \multicolumn{9}{|l|}{ Orthoptera } \\
\hline no identificados & 15.7 & 9 & 36.8 & 11.0 & 17.1 & 5.4 & 28.3 & 11.1 \\
\hline \multicolumn{9}{|l|}{ Hymenoptera } \\
\hline Formicidae & 0.7 & 0.2 & 2.9 & 0.4 & 0 & 0 & 0.3 & 0.6 \\
\hline Otras familias & 0 & 0 & 0 & 0 & 0.3 & 0.1 & 0 & 0 \\
\hline \multicolumn{9}{|l|}{ Dictyoptera } \\
\hline Blattidae & 1.5 & 0.9 & 2.9 & 0.9 & 3.7 & 1.1 & 6.6 & 2.8 \\
\hline TOTAL INSECTA & 74.5 & 26.5 & 80 & 19.1 & 81.6 & 17.6 & 61.5 & 19.4 \\
\hline \multicolumn{9}{|l|}{ Scorpionida } \\
\hline no identificadas & 14.6 & 20.7 & 10.3 & 7.8 & 4.7 & 3.6 & 0 & 0 \\
\hline \multicolumn{9}{|l|}{ Araneae } \\
\hline no identificadas & 0.7 & 0.9 & 0 & 0 & 0.3 & 0.3 & 17.5 & 17.1 \\
\hline TOTAL ARACHNIDA & 15.3 & 21.7 & 10.3 & 7.8 & 5 & 3.9 & 17.5 & 17.1 \\
\hline \multicolumn{9}{|l|}{ Decapoda } \\
\hline no identificadas & 0.2 & 0.3 & 1.7 & 1.3 & 0 & 0 & 0 & 0 \\
\hline TOTAL MALACOSTRACA & 0.2 & 0.3 & 1.7 & 1.3 & 0 & 0 & 0 & 0 \\
\hline
\end{tabular}


En cuanto a biomasa, el grupo principal fueron los mamíferos, seguido de los artrópodos y las aves (Tabla 1). Los vertebrados (aves y mamíferos) aportaron mayor biomasa en todas las parejas; de estos, los mamíferos fueron el grupo más importante (Tabla 2). No obstante, en la pareja 1 los artrópodos aportaron casi la mitad de la biomasa consumida (48.5\%).

El índice de Levins estandarizado, total y por parejas, mostró resultados menores a 0.5 . Las tres parejas de la isla Las Casitas (P1, P2 y P3) fueron más generalistas en su dieta, en comparación con la pareja 4 (P4) de la isla de Las Huacas (Tabla 3). La prueba de Kruskal-Wallis no evidenció diferencias significativas entre la dieta de las cuatro parejas estudiadas $(p=0.4931)$.

\section{Discusión}

Las presas más consumidas por $A$. cunicularia punensis en el archipiélago de Jambelí, en cuanto a frecuencia de aparición, son los artrópodos. En biomasa el grupo de mayor aporte lo componen los mamíferos, al igual que en la mayoría de los estudios realizados (Nabte 2004, Motta-Junior \& Bueno 2004, Moulton et al. 2005, Tommaso et al. 2009, Solaro et al. 2012, Cadena-Ortiz et al. 2016).

Las medidas de las egragópilas recolectadas en el archipiélago de Jambelí son similares en tamaño y peso a las reportadas por Cadena-Ortiz et al. (2016) en los Andes ecuatorianos.

Registramos una media de 15.41 presas por egagrópila, un número alto en comparación con datos previos. Por ejemplo, es un 38\% mayor al número de presas reportado por Cruz-Jofré $\&$ Vilina (2014) en otro sistema insular del norte de Chile, y entre 44-58\% mayor a otros estudios (Nabte 2004, Moulton et al. 2005, Solaro et al. 2012). En el único estudio publicado en Ecuador sobre esta especie (Cadena-Ortiz et al. 2016) la media de presas por egagrópila es apenas de 2.61 .

En la disección de las egagrópilas encontramos conchas de pequeños gasterópodos (caracoles), como en otros estudios realizados (Medina et al. 2013, Cadena-Ortiz et al. 2016). Además, encontramos en una egagrópila escamas de una serpiente de la familia Colubridae, posiblemente Oxyrhopus fritzingeri, la única especie de esta familia registrada en las islas (Sánchez \& Yánez-Muñoz 2015). Otra egagrópila contenía escamas de pez. Si bien estudios similares realizados cerca del mar también se encontraron escamas en las egagrópilas (Vieira \& Teixeira 2008, Cruz-Jofré \& Vilina 2014), es posible que este material provenga del contenido estomacal de una presa consumida por los búhos, ya que no existe evidencia actual de carrońeo o pesca por parte de $A$. cunicularia.

Algunos restos encontrados en los alrededores o en la entrada de las madrigueras no estuvieron presentes en las egagrópilas, por ejemplo Rhinella horribilis, una de las presas más abundantes en todas las madrigueras. En otros estudios de la dieta de $A$. cunicularia también aparecen sapos y ranas de manera frecuente (Motta-Junior \& Bueno 2004, Tommaso et al. 2009, Solaro et al. 2012).

Entre las defensas de los anuros para evitar la depredación están algunas secreciones tóxicas (Krishna \& Vijayalaxmi 2004), que provienen principalmente de las glándulas parótidas y los ovarios (Lever 2001); por ello, algunas aves de presa son capaces de desmembrar a estas, para alimentarse de las partes menos tóxicas del cuerpo (Beckmann \& Shine 2009). Si bien Marks et
Tabla 3. Amplitud de nicho trófico y riqueza de especies de los cuatro territorios de Athene cunicularia punenesis estudiados en el archipiélago de Jambelí, El Oro, suroeste de Ecuador. Los resultados se muestran por pareja y en general. Índice de Levins (B); Índice de Levins estandarizado (BA); Riqueza de especies (S).

\begin{tabular}{cccc}
\hline & B & BA & S \\
\hline P1 & 5.171 & 0.348 & 19 \\
P2 & 3.450 & 0.153 & 16 \\
P3 & 4.106 & 0.207 & 17 \\
P4 & 2.891 & 0.105 & 13 \\
General & $\mathbf{4 . 1 5 4}$ & $\mathbf{0 . 1 0 9}$ & $\mathbf{3 0}$ \\
\hline
\end{tabular}

al. (2017) indican que $A$. cunicularia captura anuros, pero evita su consumo al encontrarlos presumiblemente desagradables. En el archipiélago de Jambelí evidenciamos el consumo de tejidos blandos de $R$. horribilis, aunque no su cabeza y piel, donde se localizan las glándulas segregadoras de toxinas. Un comportamiento similar se ha documentado en Corvus corvus depredando a Bufo boreas (Brothers 1994) y Corvus macrorhynchus consumiendo a Bufo parietalis (Krishna \& Vijayalaxmi 2004), pero no hemos localizado información previa sobre este comportamiento alimenticio en Strigiformes.

Adicionalmente, en la entrada de cada nido se observó estiércol de cánidos y frutos del árbol Terminalia catappa (Combretaceae). König \& Weick (2008) han propuesto que este comportamiento tiene la finalidad de distraer a potenciales depredadores y evitar que los descubran por el olor. Un comportamiento similar se ha identificado en Asio flammeus, que nidifica en o cerca del suelo (König \& Weick 2008).

La mayoría de los estudios publicados, reportan como el grupo más consumido por $A$. cunicularia a los artrópodos (Nabte 2004, Motta-Junior \& Bueno 2004, Vieira \& Teixeira 2008, Tommaso et al. 2009, Solaro et al. 2012, Medina et al. 2013, Cruz-Jofré \& Vilina 2014, Cadena-Ortiz et al. 2016). En nuestro estudio, entre los artrópodos, los coleópteros son los más prevalentes, en particular la familia Tenebrionidae. Los mamíferos representan tan solo un $12 \%$ en frecuencia de aparición, pero son los que aportan mayor biomasa a la dieta de $A$. cunicularia punensis (58.5\%).

En el presente estudio, las aves representan un porcentaje muy bajo en la dieta, pero su aporte en biomasa no es tan bajo si lo comparamos con otros estudios (Andrade et al. 2004, 2009, Tommaso et al. 2009, Solaro et al. 2012, Cadena-Ortiz et al. 2016). Cadena-Ortiz et al. (2016) no reportan restos de aves en las egagrópilas, pero sí en la entrada de los nidos. Según estos autores, las aves pueden estar subestimadas cuando se realiza el estudio de la dieta mediante el análisis de egagrópilas.

Curiosamente, pese a que los saurios Dicrodon guttulatum y Microlophus occipitalis son abundantes en el archipiélago de Jambelí, ninguno de ellos apareció en las egagrópilas, al contrario que en otros estudios realizados en el país (Cadena-Ortiz et al. 2016) o en ecosistemas insulares (Cruz-Jofré \& Vilina 2014), donde sí se encontraron saurios. Esto podría estar condicionado por la presencia de Gampsonyx swainsonii en las dos islas estudiadas. Este elanio es un especialista en depredar saurios (Ferguson-Lees \& Christie 2001). Por otra parte, la ausencia de mamíferos nativos en la dieta de $A$. cunicularia punensis en 
Jambelí es notable. Los únicos mamíferos identificados corresponden a dos especies plaga introducidas, las mismas que se consideran como causantes de la extinción de roedores y otras especies nativas en varios sistemas insulares (Dowler et al. 2000, Harris \& Macdonald 2007). Es prioritario iniciar un estudio sobre las poblaciones de micromamíferos nativos en nuestra área de estudio.

En todas las parejas estudiadas, los invertebrados tuvieron la frecuencia de aparición más alta $(79-90 \%)$ y los vertebrados hicieron el mayor aporte de biomasa $(51.5$ - 78.6\%). La pareja de la isla Las Huacas consumió un mayor número de individuos de Mus musculus, con 19\% en frecuencia de aparición y más del $50 \%$ de aporte energético. Esta fue la única pareja que no consumió Rattus rattus, y presentó mayor frecuencia de aparición $(2.1 \%)$ y aporte energético (11.4\%) de aves. En cuanto a las diferencias en frecuencia de aparición de invertebrados encontradas entre nidos pueden deberse a la disponibilidad de invertebrados en cada isla. La pareja 4, que está en una isla diferente (Las Huacas), tiene la menor diversidad de artrópodos en su dieta, y por tanto, el menor índice de amplitud de nicho trófico (Tabla 3). En otros estudios en sistemas insulares (Cruz-Jofré \& Vilina 2014), el consumo de artrópodos varió muy poco entre islas $(89.2 \%-92.5 \%)$, aunque en una de ellas no aparecieron restos de artrópodos. La amplitud de nicho trófico encontrada, según el índice de Levins estandarizado, fue muy similar a otros estudios aunque ligeramente mayor al planteado por Sánchez et al. (2008) y Solaro et al. (2012) o menor por el propuesto por Tommaso et al. (2009); y varió desde 19 especies consumidas por la pareja 1, hasta 13 especies por la pareja 4 (Tabla 3). La mayor superficie de bosque seco nativo en la isla de la comunidad de Las Casitas probablemente influyó en la mayor diversidad de presas disponibles.

El presente trabajo aporta la primera información de campo sobre la subespecie $A$. cunicularia punensis en Ecuador y complementa el trabajo de Medina et al. (2013) sobre esta subespecie poco estudiada, de distribución restringida a la zona árida del suroeste de Ecuador y noroeste de Perú (Holt et al. 2017). Estudios complementarios de abundancia de presas en el archipiélago de Jambelí podrían proveer información sobre la disponibilidad y selección de presas por $A$. cunicularia punensis, así como las relaciones tróficas con otros depredadores tanto diurnos como nocturnos.

\section{Agradecimientos}

A la Universidad Técnica Particular de Loja por financiar el trabajo de campo en las islas, y a las comunidades de Las Huacas y Las Casitas por ayudarnos con la logística. A Ivonne González por la elaboración del mapa del archipiélago de Jambelí. A Carlos Íñiguez por el apoyo en el análisis estadístico.

\section{Literatura citada}

Andrade A., P. Teta \& J.R. Contreras. 2004. Dieta de la lechucita vizcachera (Speotyto cunicularia) en el parque nacional Médanos del Chaco (Paraguay). Ornitologia Neotropical 15: 87-92.

Beckmann C. \& R. Shine. 2009. Impact of invasive cane toads on Australian birds. Conservation Biology 23(6): 1544-1549. https://doi.org/10.1111/j.1523-1739.2009.01261.x

Bielefeld R.R. 2014. Florida Burrowing Owl (Athene cunicularia floridana) preys on eurasian collared-dove (Streptopelia decaocto). Florida Field Naturalist 42(3): 114-118.

Brothers D. 1994. Bufo boreas (Western Toad) predation. Herpetological Review 25(3): 117.
Bueno A.A. \& J.C. Motta-Junior. 2008. Small mammal prey selection by two owl species in southeastern Brazil. Journal of Raptor Research 42(4): 248-255. https://doi.org/10.3356/ JRR-07-37.1

Cadena-Ortiz H., C. Garzón, S. Villamarín-Cortéz, G.M. PozoZamora, G. Echeverría-Vaca, J. Yánez \& J. Brito. 2016. Diet of the Burrowing Owl Athene cunicularia, in two locations of the inter-Andean valley Ecuador. Revista Brasileira de Ornitología 24(2): 122-128.

Carevic F., E.R. Carmona \& A. Mu-oz-Pedreros. 2013. Seasonal diet of the burrowing owl Athene cunicularia, Molina 1782 (Strigidae) in a hyperarid ecosystem of the Atacama Desert in northern Chile. Journal of Arid Environments 97: 237-241. https://doi.org/10.1016/j.jaridenv.2013.07.008

Cerón C. 2013. Arbustal deciduo y Herbazal de playas del Litoral. En: Ministerio del Ambiente del Ecuador 2013. Sistema de Clasificación de los Ecosistemas del Ecuador Continental. Ministerio del Ambiente del Ecuador, Quito. Pp. 34 - 36.

Cerón C.E., W. Palacios, R. Valencia \& R. Sierra. 1999. Las formaciones naturales de la Costa del Ecuador. En: R. Sierra (ed) Propuesta Preliminar de un Sistema de Clasificación de Vegetación para el Ecuador Continental. Proyecto INEFAN/ GEF-BIRF y EcoCiencia, Quito. Pp 55-78.

Clark R.J. 1975. A field study of the Short-eared Owl, Asio flammeus, in North America. Wildlife Monographs 47: 1-67.

Cruz-Jofré F. \& Y.A. Vilina. 2014. Ecología trófica de Athene cunicularia (Aves: Strigidae) en un sistema insular del norte de Chile: ¿Posible respuesta funcional y numérica frente a Pelecanoides garnotii (Aves: Pelecanoididae)? Gayana 78 (1): 31 40. https://doi.org/10.4067/S0717-65382014000100005

Dowler R.C., D.S. Carroll \& C.W. Edwards. 2000. Rediscovery of rodents (Genus Nesoryzomys) considered extinct in the Galapagos Islands. Oryx 34(2): 109-118. https://doi. org/10.1046/j.1365-3008.2000.00104.x

Ferguson-Lees J. \& D.A. Christie. 2001. Rapaces del mundo. Editorial Omega. Barcelona, Espa-a. 1082pp.

Freile J.F. \& T. Santander. 2005. Áreas Importantes para la Conservación de las Aves en Ecuador. Aves y Conservación, BirdLife International, Conservación Internacional y Ministerio del Ambiente de Ecuador. Quito, Ecuador.

Greene C.H. 1986. Patterns of prey selection: implications of predator foraging tactics. American Naturalist Journal 128: 824-839. https://doi.org/10.1086/284608

Hall D.B., P.D. Greger \& J.R. Rosier. 2009. Regional and seasonal diet of the Western Burrowing Owl in south central Nevada. Western North American Naturalist 69(1): 1-8. https://doi. org/10.3398/064.069.0101

Harris D.B. \& D.W. Macdonald. 2007. Interference competition between introduced black rats and endemic Galápagos rice rats. Ecology 88(9): 2330-2344. https://doi.org/10.1890/061701.1

Holt D.W., R. Berkley, C. Deppe, P. Enríquez Rocha, J.L. Petersen, J.L. Rangel Salazar, K.P. Segars, K.L. Wood, E. de Juana \& J.S. Marks. 2017. Burrowing Owl (Athene cunicularia). In: del Hoyo, J., Elliott, A., Sargatal, J., Christie, D.A. \& E. de Juana, eds. Handbook of the Birds of the World Alive. Lynx Edicions, Barcelona. (retrieved from http://www.hbw.com/ node/55091 on 20 June 2017).

Hurlbert S.L. 1978. The measurement of niche overlap and some relatives. Ecology 59: 67-77. https://doi.org/10.2307/1936632

Jaksic F.M. \& C.D. Marti. 1981. Trophic ecology of Athene owls in mediterranean-type ecosystems: a comparative analysis. Canadian Journal of Zoology 59: 2331-2340. https://doi. org/10.1139/z81-312

Kavanagh R.P. 2002. Comparative diets of the Powerful Owl (Ninox strenua), Sooty Owl (Tyto tenebricosa) and Masked Owl (Tyto novaehollandiae) in southeastern Australia. In Newton I., R. Kavanagh, J. Olsen \& I. Taylor, eds. Ecology and conservation of owls. CSIRO Publishing, Collingwood, Australia. Pp. 175-191.

Krishna S. \& K. Vijayalaxmi. 2004. Bufo parietalis (Forest Toad) predation. Herpetological Review 35(2): 156-157.

König C. \& F. Weick. 2008. Owls of the world. 2nd ed. Christopher Helm, London, UK. 528PP. 
Krebs C. 1989. Ecological Methodology. Harper \& Row publishers. Inc. New York, EEUU.

Lack D. 1946. Competition for food by birds of prey. Journal of Animal Ecology 15: 123-129. https://doi.org/10.2307/1552

Lever C. 2001. The Cane Toad. The History and Ecology of a Successful Colonist. Westbury Publishing, West Yorkshire, UK.

Marks J.S., R.J. Cannings \& H. Mikkola. 2017. Typical Owls (Strigidae). En del Hoyo, J, A Elliott, J Sargatal, DA Christie \& E de Juana, eds. Handbook of the birds of the world alive. Lynx Edicions, Barcelona, Espa-a. Disponible en http:// www.hbw.com/node/52260 [Visitada el 2 de mayo de 2017].

Marti C.D. 1974. Feeding ecology of four sympatric owls. Condor 76: 45-61. https://doi.org/10.2307/1365983

Marti C.D., K. Steenhof, M.N. Kochert \& J.S. Marks. 1993. Community trophic structure: the roles of diet, body size, and activity time in vertebrate predators. Oikos 67: 6-18. https:// doi.org/10.2307/3545090

Marti C.D. 1987. Raptor food habits studies. In: Giron Pendleton B.A., B.A. Millsap, K.W. Cline \& D.M. Bird, eds. Raptor Management Techniques Manual. Hancock House Publisher, Washington, EEUU. Pp. 129-152.

Martínez D.R., R.A. Figueroa, C.L. Ocampo \& F.M. Jaksic. 1998. Food habits and hunting ranges of short-eared owls (Asio flammeus) in agricultural landscapes of southern Chile. Journal of Raptor Research 32: 111-115.

Medina C.A., W. Zelada, L. Pollack, E. Huamán \& A. Gómez. 2013. Dieta de la lechuza de los arenales, Athene cunicularia, en Trujillo y en el Cerro Campana, La Libertad (Perú). Rebiol 33(2): 99-106.

Morales M.B. 2016. Enciclopedia virtual de vertebrados ibéricos-aves: Passer domesticus. Disponible en http://www.vertebradosibericos.org/aves/pasdom.html [Visitada el 30 agosto de 2016]

Motta-Junior J.C. 2006. Relaçoes tróficas entre cinco Strigiformes simpátricas na região central do Estado de São Paulo, Brasil. Revista Brasileira de Ornitologia 14(4): 359-377.

Motta-Junior J.C. \& A.A. Bueno. 2004. Trophic ecology of the Burrowing Owl in southeast Brazil. En: Chancellor R.D. $\&$ B.U. Meyburg, eds. Raptors worldwide: proceedings of the VI world conference on birds of prey and owls. World Working Group on Birds of Prey and Owls, MME BirdLife, Budapest, Hungary. Pp. 763-775.

Moulton C.E., R.S. Brady \& J.R. Belthoff. 2005. A Comparison of Breeding Season Food Habits of Burrowing Owls Nesting in Agricultural and Nonagricultural Habitat in Idaho. Journal of Raptor Research 39(4): 429-438.

Nabte M.J. 2004. Dieta de Athene cunicularia (Aves: Strigiformes) en el nordeste de la provincia del Chubut, Argentina. Mastozoología Neotropical 11(2): 252-253
Pardiñas U. \& S. Cirignoli. 2002. Bibliografía comentada sobre los análisis de egagrópilas de aves rapaces en Argentina. Ornitología Neotropical 13: 31-59.

R Core Team. 2016. R: A language and environment for statistical computing. R Foundation for Statistical Computing, Vienna, Austria. Disponible en http://www.R-project.org/

Rau J. 2000. Métodos de análisis en ecología trófica. En: Mu-oz Pedreros A. \& J. Yá-ez, eds. Mamíferos de Chile. CEA Ediciones, Valdivia, Chile. Pp. 397-406.

Rocha R.G., E. Ferreira, Y.L.R. Leite, C. Fonseca \& L.P. Costa. 2011. Small mammals in the diet of Barn owls, Tyto alba (Aves: Stringiformes) along the mid-Araguala River in central Brazil. Zoologia 28(6): 709-716. https://doi.org/10.1590/ S1984-46702011000600003

Rodríguez-Estrella R. 1997. Nesting sites and feeding habits on the Borrowing Owl in the biosphere reserve of Mapimi, Mexico. Journal Raptor Research Report 9: 99-106.

Sánchez K.B., A.I. Malizia \& M.S. Bó. 2008. Trophic ecology of the burrowing owl (Athene cunicularia) in urban environments of Mar Chiquita Biosphere Reserve (Buenos Aires Province, Argentina). Ornitología Neotropical 19: 71-80.

Sánchez J.C. \& M. Yánez-Mu-oz. 2015. Herpetofauna. Capítulo IV: Manglares y Zona Marino Costera. En: Una Guía para Ecosistemas Andino-Costeros. Publicación Miscelánea N ${ }^{\circ}$ 7. Serie de Publicaciones MECNIN - GADPEO. Quito, Ecuador. Pp. 37-66.

Silva-Porto F. \& R. Cerqueira. 1990. Seasonal variation in the diet of the Burrowing Owl Athene cunicularia in a restinga of Rio de Janeiro. Ciência e Cultura 42: 1182-1186.

Silva S.I., I. Lazo, E. Silva-Aranguiz, F.M. Jaksic, P. Meserve \& J.R. Gutiérrez. 1995. Numerical and functional response of Burrowing Owls to long-term mammal fluctuations in Chile. Journal Raptor Research 29: 250-255.

Solaro C., M.A. Santillán, A.S. Costán \& M.M. Reyes. 2012. Ecología trófica de Athene cunicularia y Tyto alba en el cerro CurruMahuida, ecotono Monte-Wspinal, La Pampa, Argentina. Hornero 27(02): 177-182.

Tommaso D.C., R.G. Callicó Fortunato, P. Teta \& J.A. Pereira. 2009. Dieta de la Lechucita Vizcachera (Athene cunicularia) en dos áreas con diferente uso de la tierra en el centro-sur de la provincia de La Pampa, Argentina. Hornero 24(2): 87-93.

Vieira L.A. \& R.L. Teixeira. 2008. Diet of Athene cunicularia (Molina, 1782 ) from a sandy coastal plain in southeast Brazil. Boletim do Museu de Biologia Mello Leitão 23: 5-14.

Wiley J.W. 1998. Breeding-season food habits of burrowing owls (Athene cunicularia) in southwestern Dominican Republic. Journal of Raptor Research 32(3): 241-245. 